\title{
Trafic de drogue et violence en Colombie
}

\section{Daniel Pécaut}

\section{(2) OpenEdition}

Journals

\section{Édition électronique}

URL : http://journals.openedition.org/conflits/117

DOI : 10.4000/conflits. 117

ISSN : $1777-5345$

Éditeur :

CCLS - Centre d'études sur les conflits lilberté et sécurité, L'Harmattan

\section{Édition imprimée}

Date de publication : 17 octobre 1991

ISSN : 1157-996X

\section{Référence électronique}

Daniel Pécaut, "Trafic de drogue et violence en Colombie », Cultures \& Conflits [En ligne], 03 | automne 1991, mis en ligne le 31 décembre 2002, consulté le 30 mars 2021. URL : http:// journals.openedition.org/conflits/117 ; DOI : https://doi.org/10.4000/conflits.117

Ce document a été généré automatiquement le 30 mars 2021.

Creative Commons License 


\title{
Trafic de drogue et violence en Colombie
}

\author{
Daniel Pécaut
}

Parmi les pays andins dans lesquels est implantée l'économie de la drogue, la Colombie représente un cas exceptionnel. Nulle part ailleurs ne s'est enclenchée une confrontation ouverte entre les narcotrafiquants et le gouvernement, comparable à celle qui atteint son paroxysme en 1989-90 sous la forme d'une terreur qui fait des milliers de victimes.

Pourtant les élites colombiennes ont longtemps voulu considérer le trafic de drogue comme un avatar de l'"économie informelle" ou de l'"économie de contrebande" qui font partie du paysage habituel.

Nous tenterons d'analyser dans cet article comment a pu se produire une montée aux extrêmes qui ne semble rationnelle ni du point de vue des trafiquants ni de celui du gouvernement. Nous suggérerons qu'elle est, dans une large mesure, le résultat d'interférences incontrôlables entre tous les protagonistes des phénomènes de violence qui secouent à nouveau la Colombie depuis 1978, l'économie de la drogue et les intérêts divers des narcotrafiquants jouant un rôle décisif dans la production de telles interférences.

Une activité économique "informelle" parmi d'autres

L'essor de l'économie de la drogue

Pendant des années, l'économie de la drogue s'est développée en Colombie sans que cela ne suscite aucune inquiétude. Elle était pudiquement évoquée comme une nouvelle rubrique de l'économie "informelle" ou, au pire, de l'économie "illégale" mais tolérée. A partir du début des années 1970, la culture de la marijuana se diffuse dans diverses zones de la Côte Atlantique. Elle est souvent le fait de petits paysans mais certaines grandes familles traditionnelles de villes comme Santa Marta ne tardent pas à prendre part, soit à la culture elle-même, soit à l'acheminement de la production vers les ports. Des Colombiens interviennent dans les opérations de transfert vers les Etats-Unis, mais ces dernières sont le plus souvent effectuées par des Américains. Néanmoins, les revenus que la Colombie tire de cette activité à la fin des années 1970 sont 
considérables. Ils subissent vers 1984 une réduction très forte mais connaissent une certaine reprise à partir de 1987-88. La réduction s'explique par les pressions exercées avec succès par les autorités américaines sur le gouvernement de Julio Cesar Turbay pour qu'il procède à des campagnes d'éradication, par les problèmes de transport d'un produit volumineux et par la concurrence d'autres pays producteurs, dont les EtatsUnis eux-mêmes. Dès la fin des années 1970, le trafic de la coca revêt une toute autre ampleur. La Colombie n'est alors qu'un modeste producteur de feuilles de coca et de pâte-base en comparaison du Pérou et de la Bolivie. Malgré une considérable expansion des surfaces cultivées au long des années 1980, on estime qu'en 1988 la production colombienne de feuilles de coca est de 21 tonnes, ce qui reste peu face aux 111.000 tonnes fournies à la même date par le Pérou et aux 51.000 tonnes fournies par la Bolivie. L'importance de la Colombie réside avant tout, cela est bien clair, dans son rôle dans l'élaboration de la cocaïne pure et dans son transport vers les marchés consommateurs. On suppose qu'elle est responsable de l'entrée de plus de $70 \%$ de la cocaïne disponible sur le marché américain. Dans la mesure où l'essentiel des bénéfices est réalisé dans la phase de l'élaboration et, plus encore, celle de la livraison aux pays consommateurs, il en résulte une accumulation spectaculaire de revenus aux mains des réseaux de narcotrafiquants. La baisse des cours qui intervient à partir de 1983 affecte tant les cultivateurs que les trafiquants. De 680 millions de dollars en 1982, les revenus perçus par les cultivateurs colombiens tomberaient à 180 millions en 1988. De 3,7 milliards de dollars en 1982, les revenus perçus par les trafiquants passeraient à 1 milliard de dollars en 1988, ce qui équivaudrait à $20 \%$ des exportations légales. Il s'agit là des estimations les plus faibles. Certains experts proposent des estimations beaucoup plus élevées. Quelles que soient les données précises, il convient surtout de constater que les trafiquants colombiens de cocaïne parviennent à se rendre maîtres du marché américain.

Contrebande traditionnelle et réseaux modernes

On comprend que les autorités colombiennes aient pu être tentées de fermer les yeux le plus longtemps possible comme s'il ne s'agissait effectivement que d'une nouvelle modalité d'économie échappant à leurs tutelles mais ne mettant pas vraiment en cause l'ordre public.

La culture de la coca se réalise le plus souvent dans des zones de colonisation qui, traditionnellement, sont soustraites à l'emprise de l'État. Pour la première fois, dans des régions périphériques, comme le Caqueta ou le Guaviare, une production s'avère hautement rentable. Que l'afflux d'une nouvelle population de colons, parfois d'origine urbaine, s'accompagne, en l'absence de toute agence officielle, de désordres et de violence; que l'occasion soit ainsi offerte à la guérilla de s'implanter dans des territoires en faisant figure de substitut de l'État, rien de cela n'est de nature à inquiéter des gouvernements habitués depuis toujours à s'accommoder de semblables turbulences.

La contrebande est une activité tout aussi habituelle. La position géographique de la Colombie contribue à ce que se soient constitués déjà à l'époque de la colonie, des couloirs sûrs, notamment celui qui traverse le département de l'Antioquia, passant par la zone de l'Uraba, pour aboutir au Panama et celui qui traverse la Guajira vers les Caraïbes et le Venezuela. Toutes sortes de denrées continuent à emprunter ces couloirs. Il est des cas, comme celui des émeraudes, dans lesquels l'État a dû pratiquement renoncer à exercer tout contrôle sur les zones d'extraction et sur les exportations. Ce sont ainsi de véritables savoir-faire qui se sont formés. L'essor du trafic de la drogue en 
a été facilité. De nombreux cadres des réseaux ont fait leur apprentissage dans la contrebande et dans les affaires d'émeraudes. Et l'État a aisément toléré cette nouvelle sorte de contrebande.

Les narcotrafiquants se présentent avant tout comme des entrepreneurs gérant des réseaux nationaux et internationaux complexes. Il n'y a pas lieu de supposer qu ils agissent de manière coordonnée. Ce que l'on va désigner comme cartels" n'est, dans un premier moment, que l'association de divers entrepreneurs dans un même réseau. Les cartels de Medellin et de Cali sont les plus importants. Ils se répartissent la majeure partie du marché de gros américain, le premier régnant sur la Côte Ouest, le second sur New York. Mais les participants à ces cartels, et surtout à celui de Medellin, peuvent conserver une part d'autonomie. Des personnes fortunées placent parfois des capitaux dans des opérations de transport réalisées par l'une ou l'autre organisation sans se sentir liées à jamais et les petits intermédiaires nationaux ne sont pas nécessairement considérés comme des membres permanents. Les capos de Cali et de Medellin sont d'ailleurs assez prudents pour ne mettre directement sous leurs ordres qu'un personnel assez restreint, quitte à recruter des hommes de mains occasionnels dans l'ample vivier de la jeunesse défavorisée des villes. Ils ne prétendent pas davantage encadrer euxmêmes les zones de culture : même en Colombie, ils préfèrent négocier avec ceux qui exercent cette fonction d'encadrement. I1 existe de surcroît bien d'autres noyaux de trafiquants, implantés par exemple à Santa Marta, Cucuta, Bogota, Pereira, qui peuvent tantôt recourir aux réseaux extérieurs des deux principaux cartels, tantôt user de relais propres. I1 n'est cependant pas à la portée de tous de tisser les vastes et mouvants ensembles d'accords internationaux nécessaires au fonctionnement des réseaux. On sait désormais que ces accords ont impliqué des relations de toutes sortes avec des circuits de blanchiment financier bien sûr, mais également avec des autorités et des organisations privées de multiples pays d'Amérique Centrale ou des Caraïbes. La qualité $\mathrm{d}$ '"entrepreneurs" des chefs des grands réseaux renvoie donc aussi à l'aptitude à mettre en oeuvre de véritables stratégies dans les coulisses des rapports entre États.

Ce constat offre un argument supplémentaire aux gouvernements colombiens pour ne pas prendre à leur compte un problème qui met en cause bien d'autres pays, dont ceux où se trouvent les masses de consommateurs.

La politique de dédramatisation de la question de la drogue

Les gouvernements sont cependant obligés, et cela dès avant 1984, de considérer le risque que les narcotrafiquants acquièrent, au plan national, une capacité d'inf1uenee politique qui altère le fonctionnement du système politique. Ce risque revêt plusieurs aspects.

Le premier réside dans le prestige que ces nouveaux riches conquièrent auprès de certaines couches de la population. Les largesses dont certains font preuve, tel Pablo Escobar finançant des équipements collectifs ou la construction de logements dans certains quartiers de Medellin, importent moins que le spectacle des réussites foudroyantes de quelques individus partis de rien. Dans une société bouleversée en l'espace de quelques années par des brassages de populations et par l'ébranlement des valeurs traditionnelles, de larges secteurs de la jeunesse urbaine voient en eux des héros comparables à ceux des séries américaines et sont tout disposés à essayer d'imiter leurs formes d'action. Aux yeux de ce public, les narcotrafiquants paraissent souvent incarner l'esprit pionnier de ceux qui ont mis en valeur, un siècle avant, les terres de l'Antioquia ou du Valle du Cauca et, en exprimant leur propre ressentiment 
social, ils réveillent des espérances populistes qui ont été, en Colombie, constamment frustrées. D'habiles propagandistes se chargent de diffuser ces images.

Le second aspect se manifeste, du reste, par les tentatives de certains trafiquants de faire leur entrée sur la scène politique. Au début des années 1980, Pablo Escobar parvient ainsi à s'intégrer un moment dans les rangs du "Nouveau Libéralisme" qui, avec Luis Carlos Galan, cherche à moraliser la vie publique; après en avoir été publiquement expulsé en raison de ses activités, il réussit à se faire élire en 1982 comme suppléant d'un représentant libéral. Carlos Lehder, quant à lui, met sur pieds dans son département du Quindio un mouvement politique, le "Mouvement latin socialiste", qui parvient en peu de mois, en distribuant généreusement des gratifications, à rallier un électorat local considérable. C est d'ailleurs à l'occasion de ces interventions politiques que les noms des trafiquants, restés jusque là relativement ignorés, commencent à s'étaler dans la presse. Celle-ci est loin de porter condamnation sur ces nouvelles figures: l'hebdomadaire le plus prestigieux, Semana, les décrit en 1983 comme des "Robin Hood". Le "banditisme social" fait, lui aussi partie de l'histoire colombienne, et même de l'histoire récente, puisqu'il a régné dans maintes zones affectées par la Violencia entre 1955 et 1965 : les narcotrafiquants donnent l'impression de le prolonger. Il reste que ces aventures politiques tournent vite court. Les deux partis traditionnels ne sont pas habitués à laisser mettre en danger leur monopole. Ils ne peuvent surtout consentir à côtoyer trop ouvertement des personnages auxquels on impute déjà de nombreux crimes.

Le troisième aspect concerne l'expansion rapide de la corruption. Elle se fait sentir dans beaucoup des domaines de la vie colombienne. Dans ceux de la justice, de la police et, plus généralement tout ce qui a trait à l'application des lois. Dans celui des activités économiques, l'argent de la drogue irriguant largement des secteurs comme la construction, le commerce, l'agriculture. Dans celui de la politique : ne plus s'afficher officiellement aux côtés des trafiquants ne signifie pas toujours dédaigner leur concours financier. La montée en force d'un clientélisme régional de type "moderne", sorte de palliatif à la crise de crédibilité qui touche les deux partis traditionnels, bénéficie souvent de ces ressources qui jouent un rôle jusque dans les élections présidentielles. Cette aggravation de la corruption inquiète certains membres des élites colombiennes. Mais elle semble à beaucoup d'autres une manifestation inévitable et modérément dommageable d'un afflux inopiné de devises.

Ces trois réalités sont assez visibles pour que le trafic de la drogue paraisse susceptible d'avoir tôt ou tard des conséquences sur la société colombienne. I1 n'est pas non plus possible d'ignorer les moyens d'intimidation déployés par les trafiquants pour faire taire ceux qui gênent leur activité. Mais, dans le contexte de guerre entre les forces de l'ordre et les guérillas, cette violence-là ne parait seulement qu'une composante de plus de la délinquance commune. Quelles que soient ses éventuelles ramifications avec la politique ou ses relations avec la corruption de certaines institutions, il y a un large accord des responsables pour préserver cette catégorisation: procéder autrement serait susceptible d'amener l'État à se battre sur un nouveau front et donnerait l'impression de s'incliner devant les Etats-Unis. Depuis 1982, le gouvernement de Belisario Betancur consacre tous ses efforts à parvenir à un cessez-le-feu avec les guérillas. Ce n'est pas un hasard s'il a fait savoir qu'il était hostile au traité, signé avec les Etats-Unis en 1979, prévoyant la possible extradition des narcotrafiquants : cela 
relève d'un nationalisme qui a l'approbation de la gauche et du souci tactique d'éviter une politisation de la question de la drogue.

En définitive, si cette question a donné lieu, et encore timidement, à un débat public avant 1984, celui-ci a porté sur ses implications sociales et économiques. Pour empêcher une "criminalisation" dangereuse du trafic et une montée artificielle des cours, certains ont envisagé la possibilité d'une légalisation. Quant aux gouvernements, ils ont dû adopter une politique face à l'afflux des ressources de "l'économie clandestine" : en ouvrant un guichet spécial de la Banque de la république pour les convertir en monnaie locale et en proclamant à leur intention des amnisties fiscales, comme en 1984, ils ont semblé contribuer, même s'ils n'avaient probablement pas d'autres choix, à dé-dramatiser, là aussi, l'économie de la drogue.

Cette orientation relève également d'un pari sur le comportement rationnel des trafiquants : comme gestionnaires de réseaux internationaux, ils semblent avoir tout intérêt à maintenir un profil bas en Colombie afin de disposer d'une quasi-impunité. Ils n'ont rien à gagner ni à se muer en un protagoniste supplémentaire de la violence socio-politique ni à s'attaquer ouvertement au régime.

Les narcotrafiquants et la violence socio-politique

Le glissement vers la violence

Quatre événements contribueront cependant à modifier les attitudes des gouvernements et de l'opinion publique à l'égard des entrepreneurs de la drogue et à entraîner progressivement une partie de ceux-ci vers la participation délibérée à la violence sociopolitique.

En févier 1983, le Procureur général de la nation publie un rapport sur un groupe d'"autodéfense" créé en décembre 1981 par les narcotrafiquants et financé par eux : le MAS (Muerte a los sequestradores). A la suite de l'enlèvement par un groupe du M19 d'un membre de la famille des Ochoa, figures essentielles du cartel de Medellin, l'ensemble des narcotrafiquants ont mis sur pied cette organisation. Le rapport établit qu'en collaboration avec des militaires et policiers en activité ou en position de réserve, le MAS a assassiné de nombreux militants de gauche. C'est donc que les trafiquants entrent dans la voie de l'intervention politique en collusion avec des éléments des forces de l'ordre.

En 1983 et 1984, l'ambassadeur des Etats-Unis en Colombie dénonce, dans de nombreuses déclarations, tant le rôle de Cuba et du Nicaragua dans le transport de la cocaïne que la collaboration des guérillas avec les trafiquants: il lance à ce propos l'expression de "narco-guérilla". Il va de soi que cela équivaut à rejeter brutalement la classification du trafic sous la rubrique "délinquance commune" pour lui donner un statut explicitement politique. Complémentairement, du reste, cela jette le doute sur le bien-fondé des efforts de Betancur pour négocier avec les guérillas puisque ces dernières sont présentées comme associées à la délinquance internationale.

Le 30 avril 1984, le ministre de la Justice est assassiné. Au cours des mois antérieurs, celui-ci avait révélé les liens de certains hommes politiques avec les trafiquants ainsi que la pénétration des ressources de la drogue dans maints secteurs de la vie colombienne. C'est le signe que les trafiquants sont décidés à frapper à tous les niveaux s'ils se sentent menacés.

Le 6 novembre 1985, le M19 s'empare du Palais de Justice : la riposte de l'armée se solde par un massacre dans lequel périssent plus de la moitié des membres de la Cour 
Suprême de Justice. Des responsables civils et militaires font courir la rumeur que l'opération du M19 était montée avec le concours de certains trafiquants qui visaient à détruire les archives judiciaires. Rumeur sans fondement, semble-t-il, mais qui contribue cependant à accréditer l'idée que les narcotrafiquants sont devenus des protagonistes de la violence entretenant des relations diverses avec les autres protagonistes.

Ces événements sont de nature différente. Deux d'entre eux mettent effectivement en cause les trafiquants. Deux autres sont fondés sur des jugements ou des rumeurs, mais leur confèrent une soudaine visibilité comme protagonistes politiques. Il faut souligner que plusieurs responsables politiques ont voulu jusqu'au bout enrayer le glissement qui risquait de s'ensuivre. Trois semaines après l'assassinat de son ministre de la Justice, Betancur a donné son aval à des entretiens secrets à Panama entre les capos du cartel de Medellin et des personnalités comme l'ancien président Lopez Michelsen ou le Procureur général pour arriver à un nouveau modus vivendi. Cela ne freine pas la détérioration rapide de la situation. Le massacre du Palais de Justice ruine définitivement la "politique de paix" de Betancur; il ouvre surtout l'ère de la violence protéiforme caractérisée par des interférences nombreuses et souvent contradictoires entre tous les acteurs.

Dans cette phase de "guerre sale", certains noyaux de trafiquants, comme le cartel de Cali, préservent, dans une certaine mesure, un profil bas. Mais d'autres, comme le cartel de Medellin apparaissent effectivement comme des protagonistes décisifs car ils sont au centre de toutes les interférences. Nous l'avons dit: cette conversion en protagoniste majeur des phénomènes de violence semble, au premier abord, irrationnelle. Il convient d'analyser les motifs qui peuvent l'expliquer. Nous distinguerons ceux qui tiennent à la diversification des intérêts des trafiquants et ceux qui renvoient aux changements plus généraux intervenus dans le contexte social et institutionnel en relation ou non avec l'intervention des narcotrafiquants. Nous laissons provisoirement de côté ceux qui découlent des pressions des Etats-Unis.

Intérêts et stratégies de violence

Certains intérêts sont permanents : ceux qui concernent l'impunité des personnes et la sécurité des réseaux. L'usage de la violence de la part des trafiquants dépend dans ce domaine de l'attitude des autorités, de l'opinion ou de tous les acteurs susceptibles de représenter une menace.

Après l'assassinat du ministre de la Justice, les divers capos sont obligés d'entrer dans une clandestinité plus ou moins complète ou de partir à l'étranger, le gouvernement de Betancur lançant une première campagne d'envergure contre certains d'entre eux, procédant à l'arrestation de quelques trafiquants et usant même pour la première fois de l'extradition dans le cas d'individus de second rang. L'opinion commence elle aussi à devenir plus hostile. La riposte des trafiquants vise à neutraliser la justice et les médias, par l'intimidation, les assassinats ou la corruption. Il en ira de même, mais à une autre échelle, à partir de 1987 et surtout en 1989-90.

Quant à la sécurité des réseaux, elle passe ordinairement par la corruption des forces de l'ordre implantées localement ainsi que par des accords tacites avec les guérillas qui contrôlent les zones de culture ou de laboratoires clandestins. Le non-respect par un groupe des FARC de tels accords a été invoqué par Gonzalo Rodriguez Gacha pour justifier la campagne impitoyable qu'il mènera entre 1986 et 1989, au nom du cartel de Medellin, contre tous les secteurs soupçonnés de sympathie envers la guérilla. Mais le 
souci d'assurer l'acheminement de la drogue conduit également, en certaines occasions, à vouloir acquérir le contrôle de territoires bien situés, comme par exemple la vallée du Moyen Magdalena ou les ports et plages secondaires de la côte atlantique.

A mesure que les narcotrafiquants consolident leur puissance socio-économique, d'autres intérêts surgissent. Ils investissent ainsi des sommes considérables dans l'achat de terres. En 1988, on pense qu'ils possèdent au minimum un million d'hectares de terres d'excellente qualité. Dans ces conditions, ils se retrouvent avec les préoccupations des autres propriétaires: se soustraire aux impôts prélevés par les guérillas et se débarrasser des colons gênants. La différence est qu'ils disposent de moyens financiers et de contacts pour mettre sur pied des groupes d'hommes de mains. Dans de nombreux cas, il n'y a pas lieu de penser que les cartels interviennent en tant que tels pour soutenir ces initiatives: elles résultent de décisions individuelles ou locales. Dans le cas de régions où les principaux associés des cartels ont acquis de nombreuses propriétés, il en va autrement, d'autant qu'il s'agit souvent aussi de régions stratégiquement importantes. Dans le Moyen Magdalena, la zone d'Uraba ou le département du Cordoba, l'influence de capos comme Gonzalo Rodriguez Gacha est très nette. Les bandes armées qu'il finance ont d'ailleurs un statut ambigu: elles se présentent au départ comme des "groupes d'autodéfense" reconnus par la loi. De la sorte, les trafiquants acquièrent l'aspect de mafias classiques, agissant dans le cadre d'une certaine légalité et définissant les formes d'organisation de la population. Accessoirement, cela leur permet de gérer le trafic de drogue en toute tranquillité. A cette évolution concourt un autre facteur : pour agir de cette manière, les trafiquants reçoivent l'appui des forces de l'ordre locale, qui arment et conseillent les groupes d'autodéfense. L'association des forces de l'ordre, des trafiquants et des propriétaires est ce qui conduit à la constitution des bandes "paramilitaires" proprement dites. Un des centres les plus connus où opère une telle coalition est la petite ville de Fuerto Boyaca, sur le rio Magdalena, où s'ouvrent les écoles de formation avec la participation de mercenaires étrangers, israéliens et anglais. Une grande partie de la population locale, lassée des exactions des guérillas, accepte ce qui a l'allure d'une entreprise de libération. Les paramilitaires de Puerto Boyaca élargissent rapidement leur rayon d'action : ils sont à l'origine des massacres commis le long de la vallée du Magdalena et de ceux perpétrés en 1988 dans l'Uraba. Des centres semblables se créent dans d'autres régions, les plaines orientales par exemple. Sur la base de leurs nouveaux intérêts, les narcotrafiquants se transforment donc rapidement en protagonistes politiques de première importance. Ils prennent part à la campagne d'extermination des militants de l'Union patriotique, accusée de maintenir des relations avec les FARC, des leaders d'organisations populaires ou de défenseurs des droits de l'homme. Ils deviennent des intermédiaires indispensables dans la lutte contre les guérillas. Ils acquièrent le pouvoir de narguer le pouvoir. Il n'est pas sûr que cette montée en puissance politique ait répondu à un dessein prémédité. Il s'agit plutôt de l'accumulation d'actions locales qui suscitent une situation sur laquelle le gouvernement ne semble plus avoir de prise.

D'autant que ces actions prennent place dans une contexte de violence généralisée et de crise institutionnelle.

La décomposition du contexte social et institutionnel

Dès 1986-87, l'affaiblissement des appartenances et des solidarités collectives est sensible sur la majeure partie du territoire colombien. La lutte de guérilla et la violence urbaine ou rurale ne se déroulent plus sur fond d'antagonismes ni d'enjeux sociaux structurés; elles ont une dynamique propre qui accentue la déstructuration des 
rapports sociaux. Cette dynamique ne peut cependant être isolée du problème de la drogue. L'économie de la cocaïne influe en amont sur elle. La transformation des narcotrafiquants en exprime les conséquences en aval.

La multiplication des fronts de la principale organisation de guérilla, les FARC, au début des années 1980 est incompréhensible si l'on ne considère pas les ressources qu'elles tirent de l'économie de la drogue, de leur implantation dans les zones de culture et des plantes à drogue. Les colons de ces zones doivent accepter de leur verser des taxes en échange de la protection qui leur est accordée. La relation qui s'établit alors reste fragile et intéressée. En complétant leur financement par le recours de plus en plus immodéré aux enlèvements et au racket, certains fronts de guérilla finissent par donner le sentiment de se mouvoir dans le même univers de valeurs instrumentales que les trafiquants, voire de se rapprocher de la délinquance commune.

La violence ordinaire de certaines villes comme Medellin ou certaines régions rurales traduit une rage sociale diffuse, mais qui ne parvient plus à se formuler en termes de conflictualité organisée. Le modèle d'action des capos de la drogue devient la seule référence idéale au risque d'accentuer davantage les manifestations de déstructuration sociale. De surcroît, la paralysie des médiations institutionnelles et coutumières fait de la force, une composante de la vie ordinaire.

A propos de la crise institutionnelle, nous ne mentionnerons que l'exemple le plus patent, celui de la justice. Son fonctionnement n'a jamais été satisfaisant. Le blocage devient presque complet à partir de 1982-84. Il se trouvera des juges pour oser poursuivre des narcotrafiquants et le payer de leur vie. Mais l'ensemble de l'institution cesse d'intervenir même dans les affaires les plus ordinaires. La Cour Suprême de Justice paraît elle-même avant tout soucieuse de veiller sur sa propre sauvegarde : en prenant par deux fois, en 1986 et 1987, une décision qui invalide le traité d'extradition, elle prive le gouvernement d'une partie de sa légitimité dans son combat contre les narcotrafiquants. Cette carence judiciaire est le symptôme de l'effondrement de nombre de régulations. Elle laisse le champ libre à tous les protagonistes de la violence.

Interférences complexes et perte des repères politiques

Les remarques antérieures prétendent surtout mettre en lumière la complexité des interférences entre les actions de tous les protagonistes. Nous avons vu les collusions entre trafiquants, propriétaires et forces de l'ordre contre la guérilla et ses soutiens potentiels. Mais des accords tacites entre les trafiquants et les guérillas sont nécessaires pour permettre les expéditions de drogue. D'autres peuvent parfois être conclu pour réaliser des importations d'armes. Des coïncidences politiques sont éventuellement susceptibles de se produire dans des moments où il s'agit d'atteindre l'autorité du gouvernement et, naturellement, de mettre en cause la politique américaine. La gauche n'est pas en reste sur les trafiquants pour rejeter, au nom de la dignité nationale, tout usage de l'extradition.

Les interférences et les collusions engendrent simultanément es divisions parmi tous les protagonistes. Cela est vrai parmi les forces de l'ordre ou les administrations régionales en fonction des complaisances que leurs membres sont soupçonnés d'avoir avec des narcotrafiquants comme cela l'est parmi le personnel politique. Mais cela n'affecte pas moins toutes les catégories de "rebelles" ce qui donne naissance à d'innombrables affrontements à l'intérieur de chaque affrontement. A partir de 1987 éclate celui du cartel de Cali contre celui de Medellin, avec un solde de centaines de victimes. La région des émeraudes est en proie à un affrontement aussi sanglant : en 
s'associant à l'un des camps en 1987, Gonzalo Rodriguez Gacha, originaire de la région, s'efforcera vainement d'en gagner le contrôle. Ailleurs, ce sont les diverses guérillas qui se disputent des territoires en même temps qu'elles s'affrontent aux groupes paramilitaires. Telle est la situation dans le Cauca ou dans l'Uraba. A quoi il faut ajouter les combats entre les bandes de jeunes de Medellin pour dominer tel ou tel quartier. Divisions internes et fragmentation territoriale vont de pair.

Ces interférences aboutissent également à effacer les frontières entre ce qui relève du politique et ce qui n'en relève pas. Tout en restant d'abord des entrepreneurs qui ont réussi, les narcotrafiquants deviEnnent des protagonistes politiques. Tout en demeurant des acteurs politiques, les guérillas donnent leurs cautions à toutes sortes de moyens de la délinquance ordinaire et se contentent d'offrir à leurs combattants une manière de vivre. Le brouillage des délimitations précises permet d'ailleurs à maints individus de circuler d'un "secteur d'activité" à l'autre, les guérillas fournissant en particulier beaucoup de cadres aux groupes paramilitaires. Il n'est pas surprenant que la logique utilitariste semble souvent l'emporter sur toute autre. En effet, tant les succès des trafiquants que la déstructuration des régulations sociales y concourent, comme si l'utilitarisme fournissait à tout le moins, des critères de rationalité pour faire face à l'urgence.

Le moment de la montée aux extrêmes

Il n'est pas dans notre propos de décrire le déchaînement sans précédent de terrorisme qui intervient entre août 1989 et juin 1990, faisant à son tour des centaines de victimes et frappant des dirigeants politiques de premier plan, dont. Trois candidats présidentiels. Une telle "guerre", pour reprendre le terme utilisé, tant par les narcotrafiquants qui y sont impliqués que par le gouvernement de Virgilio Barco, est très différente des phénomènes de violence auxquels nous venons de nous référer : la fragmentation des scènes conflictuelles et des enjeux cède la place à une confrontation directe se livrant autour de la seule question de l'extradition et qui en arrive à menacer un moment le régime. Ce nouveau glissement est à bien des égards surprenant. Comme son prédécesseur, le gouvernement Barco s'est au départ efforcé de traiter le conflit comme s'il s'agissait de contenir des actions relevant de la délinquance commune et a voulu notamment éviter de mettre en oeuvre des mesures d'extradition pour préserver une possibilité de transaction avec les trafiquants.

Il est une première manière d'expliquer cette soudaine montée aux extrêmes. Celle-ci serait liée à l'internationalisation du problème de la drogue, les pressions américaines réduisant la marge de manoeuvre du gouvernement colombien et amenant les "extraditables" à recourir au terrorisme à grande échelle. Le fait même que l'extradition devienne finalement le principal enjeu semble confirmer cette hypothèse. De surcroît, la façon dont sont désignés les "extraditables" va dans le même sens. Tous les grands narcotrafiquants ne sont pas également affectés par cette étiquette : ce sont avant tout ceux qui gèrent les contacts avec des États ou des réseaux étrangers divers. Les Etats-Unis ne dénoncent plus seulement le "narco-terrorisme" mais la subversion internationale. Le gouvernement colombien est de la sorte incité à recourir à la seule force, déclenchant par là une riposte dont l'intensité n'était pas prévisible.

II faut cependant prendre en compte simultanément des facteurs internes. Lorsque le gouvernement de Barco se prépare à l'affrontement direct, avant l'assassinat de Luis Carlos Galan qui se produit à l'instant même où est divulgué un train de mesures répressives, il est en danger de perdre totalement prise sur la situation. Certains 
membres des secteurs de force de l'ordre, de la classe politique et des trafiquants parait susceptible de provoquer une brutale désagrégation de l'État. La prolifération des groupes paramilitaires échappe à tout contrôle. II est du reste vite évident que Virgilio Barco ne dispose pour livrer la "guerre" que de l'appui effectif de forces réduites et d'une fraction très minoritaire des leaders politiques. A la fin de 1989, en dépit de la mort de Gonzalo Rodriguez Gacha, seul résultat tangible de la campagne contre le cartel de Medellin, Virgilio Barco semble presque totalement isolé. La conjonction du terrorisme des trafiquants et de l'offensive de plusieurs organisations de guérilla, en avril 1990, met un moment en question le déroulement de l'élection présidentielle. Les interférences entre tous les protagonistes de la violence font peser un doute sur l'origine de certains des assassinats les plus spectaculaires comme ceux de Bernardo Jaramillo Ossa, président de l'Union patriotique ou de Carlos Pizarro qui a dirigé la conversion du M19 en parti politique légal. La confrontation secoue en définitive l'État autant que la société. L'enjeu pour Barco et son entourage est de préserver ce qui reste d'autorité à l'État.

\section{Conclusion}

La désescalade qui se produit dès l'entrée en fonction de Cesar Gaviria peut donner l'impression que le gouvernement Barco a provoqué lui-même cette montée aux extrêmes sans issue. Ce n'est pas si simple. Il a largement créé simultanément les conditions rendant possible une transaction implicite.

Une de ces conditions est que l'État colombien retrouve, aux yeux des pays étrangers comme de l'opinion interne, une certaine autorité. La "guerre" de Barco permet au gouvernement de Gaviria de chercher un compromis sans paraitre abdiquer. Il peut se réclamer de la souveraineté nationale pour traiter un conflit interne sans que les EtatsUnis ou d'autres États puissent s'indigner eu égard au prix payé au préalable par la Colombie. Un second facteur est l'affaiblissement des "extraditables". La transformation en protagonistes politiques déclarés a montré ses conséquences négatives. Le retour à un profil bas apparaît à nouveau comme rationnel par rapport aux intérêts directs. La dernière circonstance consiste en ce qu'une possibilité s'offre d'insérer, l'air de rien, la transaction avec les trafiquants à l'intérieur d'une vaste entreprise d'"ouverture" politique. Le succès du M19 comme force politique, puis le ralliement de plusieurs autres organisations de guérilla à la légalité confèrent à la convocation une Assemblée constituante au premier semestre de 1991 l'aspect d'une vaste entreprise de réconciliation entre les Colombiens et de modernisation politique. Nul ne trouve à redire lorsque cette Assemblée se prononce contre l'extradition. D'une certaine manière, les trafiquants obtiennent le traitement politique qu'ils réclamaient, mais par l'intermédiaire d'acteurs disposant d'une légitimé incontestée.

Pour autant, les gouvernements colombiens ne sont probablement pas au bout de leurs peines. Ils reviennent à la case départ, celle où la question de la drogue est posée avant tout en termes économiques ou en termes de délinquance. 


\section{RÉSUMÉS}

Après une analyse de l'économie de la drogue, économie informelle parmi d'autres, l'auteur s'interroge sur la montée spectaculaire de la violence socio-politique liée au trafic. Cette violence n'apparaît rationnelle ni du point de vue du gouvernement, ni de celui des trafiquants. I1 conclut que cette escalade est dans une large mesure produite par des interférences non contrôlées entre les différents acteurs.

Following an analysis of the economic aspect of the drug market, an informal economy among others, the author questions the reasons for the dramatic growth of socio-political violence associated to the drug traffic. From both sides, government as well as drug suppliers, this violence cannot be understood from a rational point of view. According to the author this increase is widely due to non-controlled interferences between the different actors.

\section{INDEX}

Index géographique : Amérique du Sud, Colombie

Mots-clés : contrebande et trafic, drogue, forces paramilitaires, violence

Thèmes : FARC-EP

\section{AUTEUR}

DANIEL PÉCAUT

Directeur d'Etudes à l'Ecole des Hautes Etudes en Sciences Sociales 